Editorial:

\title{
Biological Aging Research in Canada: Past, Present and Future
}

\section{Introduction}

Founded in 1971, the Canadian Association on Gerontology (CAG) is a national educational and scientific association whose mission is to develop theoretical and practical understanding of aging through multidisciplinary research, practice, education and policy analysis in gerontology, with the final aim of improving the quality of life of elderly Canadians. For the past six years I have served as the Biology Editor of the Canadian Journal on Aging which is the major vehicle for the publication of aging research in Canada and a major voice for the CAG. This position has allowed me to become relatively well-acquainted with the efforts of many of the research scientists within Canada whose work deals directly with the processes of biological aging. In this review I shall introduce the broad concept of biological aging, describe a subset of the basic science research thrusts in biological aging being undertaken in Canadian institutions and conclude with a short commentary on the future of aging research in Canada. Since this area is broad, I shall apologize in advance for the necessary exclusion of the many interesting areas of aging research that I shall not be able to mention due to length constraints.

\section{Biological Aging}

It is reasonable to assume that individuals have pondered their own mortality ever since the evolution of human abstract thought. However, it was not until the late 1800 s that a framework was established that attempted to define the process of biological aging as an event unique to multicellular organisms. In an essay published in 1891, August Weismann contended that death was not an essential attribute of living matter. $\mathrm{He}$ proposed that natural death only occurred in multicellular organisms as a consequence of evolutionary separation of the reproductive germ (egg and sperm) cells and the somatic cells which make up the vast majority of cells in higher organisms (1). While bacteria, yeast and many other single-celled organisms are functionally immortal, experiments described in the late 1950 s by Sonneborn and others have shown that some strains of unicellular Paramecium appear to be immortal while others are not (2). Thus, it appears that the phenomenon of biological aging (or senescence as it is often referred to) evolved in single-celled organisms even before the separation of germ-line and somatic cells in multi-cellular organisms. Recent 
studies have also indicated that cells within the body age at individual rates (3), that are possibly a consequence of environmental factors including pressures to replicate (4).

Contemporary biological aging research is carried out using a large array of unicellular and multicellular experimental organisms, including short-lived worms and flies and individual somatic cells of higher eukaryotes that are grown independently of the host organism in artificial medium. Cells isolated and grown in this manner outside the body (in vitro: literally "in glass") appear to remember how old they are as measured by the number of times they can divide, independently of the other cells in the body (5). Combined with studies showing that cells isolated from shortlived species divide fewer times in vitro than cells derived from long-lived species (6), such observations have prompted many researchers to examine how the processes of cellular aging might affect organismal aging of individuals within a population, and have contributed to the idea that aging has a definite genetic component. The nature of the genetic component of aging and the effects of environmental factors, such as radiation and metabolic byproducts, upon the faithful processing of messages encoded by genes is a major focus of aging research in many laboratories in Canada as described below.

\section{Genetics of Aging in a Lower Organism}

It has been over 70 years since the first scientific data were published to support the view that heredity influences human lifespan (7). Since it has proven possible to select for longevity in lower organisms that have relatively short lifespans, such as fruit flies and roundworms, these organisms have been used to try to identify specific genes involved with regulating lifespan, particularly in the nematode roundworms. Some of the genes identified that affect the lifespan of the nematode C. elegans appear to be conserved between worms and vertebrates, including humans, suggesting that they may play similar roles in different species, but whether the analogous genes in vertebrates also influence lifespan is unknown. Three classes of genes (called age-1, daf-2 and clk-1) have been cloned that, when mutated, increase lifespan but they also affect many other aspects of development and behaviour. The age-1 gene encodes a homologue of the phosphatidylinositol 3-kinase large subunit which likely plays a role in allowing the cell to respond to its environment. The daf- 2 gene encodes an insulin receptor family member that functions in cell signalling and serves to maintain correct sugar/energy levels in the cell, although clk-1 encodes a protein of unknown biochemical function. The laboratory of Dr. Sigfreid Hekimi at McGill University in Montreal was responsible for cloning four different "clock" genes (clk1-4) that very strongly affect the maximum lifespan of C. Elegans (8). Dr. Hekimi's group has provided evidence for the evolutionary conservation (and perhaps, therefore, shared function) of 
some clk genes that appear to affect the timing of physiological processes in the roundworm. Future work will address the extremely interesting questions of how mutation of these genes in higher organisms, such as mice, affects lifespan and other aspects of vertebrate growth and development.

\section{At the Cell Level, Aging is not Death}

Two major processes that are thought to limit the emergence of immortal human cancer cells are cell senescence and programmed cell death, the latter of which is also referred to as apoptosis. Apoptosis is widely believed to eliminate developing cancer cells that have sustained genetic or epigenetic damage and so loss of such a mechanism would promote the survival of mutated, and potentially tumorigenic cells in the body.

Just such a mechanism may exist in some human cell types as reported by Dr. Eugenia Wang's laboratory at the Bloomfield Center for Research in Aging in Montreal (9). Senescent fibroblasts appear to resist apoptosis, and, although the mechanism(s) by which this occurs is(are) not fully understood, it(they) may involve constitutive expression of an apoptosis survival factor called bcl2. A reduced propensity of cells to undergo apoptosis might be expected, therefore, to contribute to increased susceptibility to developing age-dependent diseases, including cancers of various types. How apoptosis and normal cell senescence affect each other will be a topic of considerable interest as the pathways regulating these processes are uncovered.

\section{The Cost of Cell Metabolism}

Senescence, as historically defined by Dr. Alex Comfort, "is a deteriorative process. What is being measured, when we measure it, is a decrease in viability and an increase in vulnerability ... . Senescence shows itself as an increasing probability of death with increasing chronological age". Chemically active free radicals are continuously generated by normal metabolic processes within cells and are usually cleared by specialized enzyme systems. Dr. Jack Carlson at the University of Waterloo is particularly interested in the possible consequences of the loss or reduction of activity in naturally occurring antioxidative systems within cells including the cytochrome P450 system (10). Many reports indicate that the levels of free radicals increase during aging and that higher levels of the enzymes that protect cells from free radicals correlate with longer lifespan. In fact, an informative study in which the protective enzymes SOD and Catalase were produced at higher levels in the fruit fly Drosophila reported that fly lifespan can in some cases be increased by up to one third (11) suggesting that loss of enzyme activity might be a causal factor in aging in this system. Aging changes seen in whole animals may be a function of free radical 
damage in particular cell types, as proposed by Dr. Carlson, and others since some cells of the body, notably nerve cells within the hypothalamus that regulate pituitary gland function might, metabolically, be particularly sensitive and important targets of free radicals.

\section{The Role of Tumour Suppressors at the Cancer: Aging Interface}

Cells cultured in vitro lose their proliferative potential after a characteristic number of cell doublings in a process termed replicative cellular senescence (5). Many reports using different experimental systems support the idea that the products of growth inhibitory tumour suppressor genes play roles in the establishment and maintenance of the state of cellular senescence. Well known examples include the increased activity or constitutive activation of the $\mathrm{Rb}$ and p53 tumour suppressors $(12,13)$. This is particularly relevant since p53 activates the expression of a number of growth inhibitors which are known to be expressed at high levels in senescent cells. Additional proteins that inhibit the initiation of DNA synthesis in early passage fibroblasts and that link the action of tumour suppressors with the cell cycle machinery, are also expressed at higher levels in senescent cells. For example, the increased expression of the tumour suppressor and cyclin-dependent kinase inhibitor p16 (14) likely contributes to the growth arrested phenotype of senescent cells. In addition to characterizing the expression and activity of known tumour suppressors, Dr. Karl Riabowol's laboratory at the University of Calgary has identified a novel candidate tumour suppressor (15). This candidate tumour suppressor (called ING1 for Inhibitor of Growth 1) appears to directly affect replicative aging since blocking its expression significantly lengthens the proliferative lifespan of normal human cells (16). Understanding how this class of potent growth regulators called tumour suppressors exert their effects in cells and interact with other regulatory molecules leaves open the exciting possibility of increasing the proliferative lifespan of key cell types in older persons. Increased cell lifespan would have obvious impacts upon such central processes as wound healing and immune response, both of which are well known to decline as the proliferative ability of fibroblasts and haematopoietic cells decrease, respectively.

\section{Blood Cell and Immune System Aging}

Blood $\mathrm{T}$ cells which play a key role in maintaining the effectiveness of the immune system are expanded and matured in the thymus. Every mammalian thymus normally undergoes a genetically programmed, marked and predictable shrinkage (involution). Involution begins soon after the first year of birth, and continues progressively throughout the entire lifespan, during which cell loss ranges from 1-5 per cent annually, slowing in later life. There is now good evidence that the blood cells remaining in 
the thymus, and other replicating cell types experience cellular aging as evidenced by the loss of telomere sequences as first described by Dr. Calvin Harley's group (17), formerly at McMaster University in Hamilton and now at Geron corporation in California. Harley and co-workers observed a progressive shortening of the ends of chromosomes (telomeres), due to loss of terminal repeat elements with each round of chromosomal replication throughout their proliferative lifespan. This loss of telomeric DNA repeats may therefore provide a genetic clock or counting mechanism that halts cell proliferation when the loss of telomeric DNA initiates a growth inhibitory signal cascade or interferes with the expression of genes at chromosomal termini. Dr. Peter Landsdorp and his group at the University of British Columbia in Vancouver have shown that even cells that divide to form all other blood cells and that are believed to be self-renewing, lose telomere sequence, have a reduced capacity to replicate and have lower levels of the enzyme telomerase that replaces telomere sequence (18). They have further shown that telomere length varies between chromosomes and that one chromosome, number 17, appears to lose telomeric sequence much more rapidly than other chromosomes (19). It will be especially interesting to see whether the length of telomeric sequence on chromosome 17 is sufficiently short to activate a hypothetical signal from the chromosome to link telomere loss with the activation of tumour suppressors such as p53 $(12,13)$.

\section{Summary}

The mechanisms responsible for biological aging have been pondered for centuries and more recently studied by a small groups of philosophers and researchers; however the amount and general quality of biological aging research increased substantially in the mid 1970s due to the formation of the National Institute on Aging in the United States in May 1974 (20). This increased quality and quantity of research in the U.S. has been a direct result of increased funding to support research in this area that will affect the vast majority of individuals in the population. No such institute exists as yet in Canada, and the overall level of funding for research, and particularly for aging research in this country remains well below the levels in other industrialized countries, including all of the G7 group (21). Despite this severe and chronic underfunding of biomedical research, many laboratories throughout Canada, including those mentioned above, manage to undertake world-class research into the basic mechanisms of biological aging. Such research should be very cost effective since it is well-established that a substantial number of ailments such as cardiovascular disease, various forms of dementia such as Alzheimer's disease and particularly, very many different cancer types (22), all are highly age-related. With the imminent greying of the Canadian population, it is hoped that the Ministers responsible for setting policy for the funding of biomedical 
research will recognize the cost effectiveness of increasing the currently substandard research budget (currently at less than $1 \%$ of health care expenditures), perhaps in the context of the proposed Canadian Institutes for Health Research. We must recognize that in light of our similar standard of living and per capita economies, and the perceived advantages of the Canadian health care system compared to the U.S. system, the aging population of Canada also deserves to reap the benefits of a vigorous, domestic biomedical aging research community rather than purchasing such benefits from our US counterparts at some later date.

\section{References}

1. Weismann, A. Essays upon heredity and kindred biological problems. London and New York: Oxford Univ. Press (Clarendon), 1891.

2. Sonneborn, TM, Rofolko, M. Aging in the P.aurelia-multimicronucleatum complex. J. Protozool. 1957; 4:21.

3. Dimri, GP, Lee, X, Basile, G, Acosta, M, Scott, G, Roskelly, C, Medrano, EE, Linskens, M, Rubeli, I, Pereira-Smith, O, Peacock, M, Campisi, J. A biomarker that identifies senescent human cells in culture and in aging skin in vivo. Proc. Natl. Acad. Sci. USA 1995; 92:9363-9367.

4. Bestilny, LJ, Gill, MJ, Mody, C, Riabowol, KT. Accelerated telomere loss implicates premature aging of the immune system in the pathogenesis of HIV infection. Submitted, 1998.

5. Hayflick, L. The limited in vitro lifespan of human diploid cell strains. Exp. Cell Res. 1965; 37:614.

6. Martin, GM, Sprague, CA, Epstein, CJ. Replicative life-span of cultivated human cells: Effects of donor+s age, tissue and genotype. Lab. Invest. 1970; 23:86.

7. Pearl, R, Pearl, R. de W. The ancestry of the long-lived. London: H. Milford, 1934.

8. Lakowski, B, Hekimi S. Determination of life-span in Caenorhabditis elegans by four clock genes. Science. 1996; 272:1010-3.

9. Wang E. Senescent human fibroblasts resist programmed cell death, and failure to suppress bcl2 is involved. Cancer Research. 1995; 55:2284-92.

10. Carlson, JC, Riley, JC. A consideration of some notable aging theories. Exp. Gerontol. 1998; 33:127-34.

11. Orr, WC, Sohal, RS. Extension of lifespan by overexpression of superoxide dismutase and catalase in Drosophila melanogaster. Science 1994; 263:1128.

12. Atadja, P, Wong, H, Garkavtsev, I, Veillette, C, Riabowol, K. Increased activity of p53 in senescing fibroblasts. Proc. Natl. Acad. Sci. USA 1995; 92:8348-8352.

13. Vaziri, H, West, MD, Allsopp, RC, Davison, TS, Wu, YS, Arrowsmith, CH, Poirier, GG, Benchimol, S. ATM-dependent telomere loss in aging human diploid fibroblasts and DNA damage lead to the post-translational activation of p53 protein involving poly(ADP-ribose) polymerase. EMBO J. 1997; 16:6018-33.

14. Wong, H, Riabowol, K. Expression of cell cycle-regulatory genes in aging human fibroblasts. Exp. Gerontol. 1996; 31:311-325. 
15. Garkavtsev, I, Kazarov, A, Gudkov, A, Riabowol, K. Suppression of the novel growth inhibitor p33ING1 promotes neoplastic transformation. Nature Genetics 1996; 14:415-420.

16. Garkavtsev, I, Riabowol, KT. Extension of the replicative lifespan of human diploid fibroblasts by inhibition of the p33ING1 candidate tumor suppressor. Mol. Cell Biol. 1997; 17:2014-2019.

17. Harley, C, Futcher, B, Greider, C. Telomeres shorten during ageing of humanáfibroblasts. Nature 1990; 345:458-460.

18. Lansdorp, PM. Telomere length and proliferation potential of hematopoietic stem cells. J. Cell Science. 1995; 108:1-6.

19. Martens, UM, Zijlmans, JM, Poon, SS, Dragowska, W, Yui, J, Chavez, EA, Ward, RK, Lansdorp, PM. Short telomeres on human chromosome 17p. Nature Genetics. 1998; 18:76-80.

20. Strehler, BL. Time, cells and aging. 2nd edition. New York, San Francisco, London: Academic Press, 1977.

21. Palca, J, Anderson, A. Science in Canada: Underinvestment lies at the core. Nature 1988; 333:719.

22. Balducci, L, Lyman, GH, Ershler, WB. (Editors). Comprehensive geriatric oncology. Amsterdam: OPA, Harwood Academic Publishers, 1998.

Karl Riabowol 